IOS Press

\title{
Review
}

\section{The Amphetamine Induced Rotation Test: A Re-Assessment of Its Use as a Tool to Monitor Motor Impairment and Functional Recovery in Rodent Models of Parkinson's Disease}

\author{
Anders Björklund ${ }^{\mathrm{a}, *}$ and Stephen B. Dunnett ${ }^{\mathrm{b}}$ \\ a Department of Experimental Medical Science, Wallenberg Neuroscience Center, Lund University, Sweden \\ ${ }^{\mathrm{b}}$ Department of Biosciences, Cardiff University, UK
}

Accepted 5 December 2018

\begin{abstract}
Rats and mice with unilateral damage to the nigrostriatal dopamine system-induced by neurotoxins, such as 6-hydroxydopamine, overexpression of $\alpha$-synuclein, or injections of toxic synuclein protofibrils-are widely used as experimental models to mimic the loss of dopamine neurons seen in Parkinson's disease. The amphetamine rotation test is commonly used to monitor the extent of motor impairment induced by the lesion, and this test has also become the standard tool to demonstrate transplant-induced functional recovery or the efficacy of neuroprotective interventions aimed to preserve or restore DA neuron function. Although the amphetamine-induced rotation test is highly useful for this purpose it has some important pitfalls and the interpretation of the data may not always be straightforward. Unless the test is applied properly and the data are displayed and interpreted appropriately the conclusions may be misleading or simply totally wrong. The purpose of this review is to draw attention to the potential problems and pitfalls involved in the use of drug-induced rotation tests, and to provide recommendations and advice on how to avoid them.
\end{abstract}

Keywords: Motor behavior, rotation, dopamine, nigrostriatal system, 6-hydroxydopamine, $\alpha$-synuclein, rat, mouse

Unilateral lesions of the nigro-striatal dopamine (DA) system induce a profound asymmetry in motor performance. In rodents, this is expressed as an asymmetric body posture, impaired use of the contralateral forelimb, and a sensori-motor orientation defect, i.e., loss of orientating movements elicited by sensory stimuli applied to the body contralateral to

\footnotetext{
${ }^{*}$ Correspondence to: Anders Björklund, Wallenberg Neuroscience Center, BMC A11, 22184 Lund, Sweden. Tel.: +46 462220540; E-mail: anders.bjorklund@med.lu.se.
}

the lesion. Since near-complete bilateral lesions of the DA projections lead to severe and long-lasting adipsia and aphagia, lesions applied to one side of the brain have been the preferred choice in experimental studies and a standard experimental model of DA deficiency similar to that seen in Parkinson's disease (PD). The unilateral lesion model is attractive in that it allows assessment of DA neuron loss and associated functional changes (impairment or recovery) on the lesioned side in direct comparison with the intact system on the non-lesioned side. 
Over the years, a range of behavioural tests have been developed to monitor impairment and recovery of motor function in rats and mice with unilateral nigrostriatal damage induced by neurotoxins, such as 6-hydroxydopamine (6-OHDA), by overexpression of $\alpha$-synuclein, or injections of toxic synuclein protofibrils. The most informative tests are those that allow quantification of spontaneous motor or sensorimotor functions (see $[1,2]$ for review). However, since such tests are relatively time-consuming and may require a period of training or shaping to the test apparatus, as well as trained personnel to perform the tests in a consistent manner, the alternative tests using drug induced rotational behavior, and the amphetamine (AMPH)-induced rotation test in particular, has become a widely used standard in the field (see [3], for review).

The AMPH-induced rotation test is attractive in that it is quick and easy to apply. It has become popular and widely used due to the fact that it can be performed by unskilled staff using automated equipment that allows testing of groups of animals at the same time, and that it allows quantification of performance in a single number. Although AMPH-induced rotation reflects the imbalance in DA release between the denervated and the non-denervated striata, it has some important pitfalls and the interpretation of the data may not always be straightforward. As a result, data obtained in this test are far too often misinterpreted or presented in a misleading way. This is important not least in cell transplantation and neuroprotection experiments where the AMPH rotation test is commonly used to demonstrate transplant-induced functional recovery or the efficacy of neuroprotective interventions to preserve or restore DA neuron function. Unless the test is applied properly and the data are displayed and interpreted appropriately, the conclusions may be misleading or simply totally wrong.

In this commentary we discuss the potential problems and pitfalls involved in the use of the druginduced rotation tests, and provide recommendations and advice on how to avoid them.

\section{HISTORY}

The pioneering work that led to the development of the rotation test was performed in the 1960s in the laboratories of Arvid Carlsson and Nils-Åke Hillarp in Göteborg and Stockholm, respectively. Using the newly introduced Falck-Hillarp histofluorescence method, Andén et al. $[4,5]$ were the first to identify the nigro-striatal DA projection in the rat, and in a paper published in 1966 [6] they went on to report on the functional effects seen after unilateral damage to the nigro-striatal pathway (NSP), induced by an electrolytical lesion placed in the cerebral peduncle. The most striking observation in this study was the vigorous ipsilateral turning behavior elicited by release of DA in the striatum on the intact side, induced by treatment with a combination of reserpine and a monoamine oxidase inhibitor.

This finding stimulated Urban Ungerstedt and Gordon Arbuthnott (working in Hillarp's former department in Stockholm) to develop drug-induced turning behavior into a reliably quantifiable functional test. In their 1970 paper [7], which has become the standard reference for this test, they took advantage of the newly introduced 6-OHDA neurotoxin as a tool to induce selective damage to DA neurons when applied unilaterally into the nigrostriatal bundle. In addition, Ungerstedt and Arbuthnott devised a purpose-built apparatus, a "rotometer", that made it possible to monitor turning simultaneously for 6 rats in spherically shaped Perspex bowls. Untreated 6-OHDA lesioned rats displayed a burst of ipsilateral turning that subsided after 3-5 minutes when placed in the rotometer bowl. However, after treatment with the dopamine stimulant drug, amphetamine, administered in doses of $1-5 \mathrm{mg} / \mathrm{kg}$, i.p., the rats turned vigorously in circles, in the direction away from the non-lesioned striatum, reaching a peak rate at about 40-60 min after injection and lasting for at least 3 hours [8]. The rotometer apparatus allows this turning to be recorded automatically as $360^{\circ}$ turns in either direction, subdivided into discrete time intervals, and is most commonly presented as either the total net turns or as the mean net turning rate (in turns/min) over the full test period (typically $90 \mathrm{~min})$.

AMPH-induced rotation has later been adapted for unilaterally lesioned mice (see e.g., $[9,10]$ ), but as discussed in a previous review [3], the automated test can be more tricky to apply to mice due to their small size and their ability to escape from any harness or jacket that may be used in the mouse-adapted rotometers.

The test is equally useful to assess the magnitude of damage to the nigrostriatal system in $\alpha$-synuclein based models of dopamine deficiency, induced by overexpression of $\alpha$-synuclein or toxic synuclein protofibrils, which have been developed to better 
reflect the pathogenesis of PD. So far, however, the extent of motor impairment obtained in these models has been highly variable and in most cases insufficient to induce robust changes in the AMPH rotation test $[11,12]$. Thus, the 6-OHDA lesion models remain the preferred and most reliable tools for the study of the functional sequelae of nigrostriatal damage in rats and mice.

\section{DRUGS USED TO INDUCE ROTATION}

Drugs used to elicit rotation are generally of two kinds. Amphetamines (AMPH; d-amphetamine or meth-amphetamine) and cocaine act pre-synaptically to stimulate DA release and/or block DA reuptake. In the absence of corresponding DA stimulation on the lesioned side, the higher concentration of released dopamine in the intact striatum results in rotation in the direction ipsilateral to the lesion [13]. By contrast, DA receptor agonists, such as apomorphine (APO), act post-synaptically and as a result of hyperstimulation of supersensitive DA receptors in the denervated striatum [14] induce rotation in the opposite contralateral direction, i.e., away from the lesioned side. Contralateral rotation is seen also after administration of L-DOPA $[14,15]$, probably due to the fact that the response elicited by DA produced from L-DOPA is amplified in the presence of supersensitive receptors on the denervated side.

$\mathrm{d}$-Amphetamine and apomorphine have become standard tools in experimental studies in rodents and they are often used in parallel. But, as we discuss further below, the scores obtained in the two tests are poorly correlated. Thus, a high rotation rate in one test does not predict a similar high rotation score in the other test. This discrepancy can be puzzling and highlights the fact that the mechanism(s) driving rotation is not fully known. The turning response induced by the two drugs is clearly non-physiological in its intensity and phenotype. Moreover, the way the lesioned rats and mice turn in response to activation of normo-sensitive receptors in the intact striatum (by amphetamine) and supersensitive receptors in the denervated striatum (by apomorphine) is distinctly different. Turning induced by amphetamine is characterised by movement in wide circles, which is in sharp contrast to the twisted body posture and tight pivoting movements induced by apomorphine, suggesting the engagement of different signaling pathways and anatomical circuits in the brain $[3,8,16]$.

\section{AMPH-INDUCED ROTATION AS A PREDICTOR OF NIGRAL DA NEURON CELL LOSS AND MOTOR IMPAIRMENT IN 6-OHDA LESION MODELS OF PD}

The AMPH rotation test is commonly used to assess the magnitude of nigral DA neuron cell loss in rats and mice. Although the rate of rotation to a given dose is determined by the asymmetry of drug-induced DA release in the two hemispheres, it is important to remember (i) that it is only an indirect and rather imprecise measure of DA neuron integrity; (ii) that the AMPH-induced turning rate is primarily determined by the degree of denervation of the dorsolateral sector of the caudate-putamen, which means that the extent of denervation in other parts of the striatum may not contribute to the same extent; (iii) that it is not exclusively determined by loss of innervation in the dorsal striatum, i.e., the caudate-putamen, but may be influenced also by the presence or absence of DA innervation in the ventral striatum, nucleus accumbens in particular (see below); (iv) that it correlates poorly with the alternative measure of turning behavior induced by DA agonists; and (v) that the motor asymmetry detected by AMPH rotation is unreliable as a stand-alone measure of motor impairment determined in tests of spontaneous motor behavior. We elaborate on each of these points in further detail below.

\section{AMPH rotation as a predictor of DA neuron integrity}

Over the years, a number of studies have been performed to validate the AMPH rotation test in rats and mice. In (Figs. 1 and 2), we summarise data from seven studies aimed to determine the relationship between the rate of AMPH-induced turning (expressed as mean turns/min over the test period) and the severity of the lesion in 6-OHDA lesioned rats and mice, expressed either as reduction in DA concentration in the lesioned striatum (Fig. 1A, D), loss of tyrosine hydroxylase $(\mathrm{TH})$ positive neurons in the substantia nigra (Figs. 1B, E and 2A-C, E, $\mathrm{H}$ ), or loss of TH-positive innervation in the ipsilateral striatum (Figs. 1C and 2D, F, G). These studies were performed in animals subjected to 6-OHDA lesions made by toxin injections either into the dorsal striatum (Figs. 1A-C and 2A), into the substantia nigra (Fig. 2B-D), or into the medial forebrain bundle (MFB) (Figs. 1D, E and 2E-H). 


\section{Intrastriatal 6-OHDA lesions in rats}

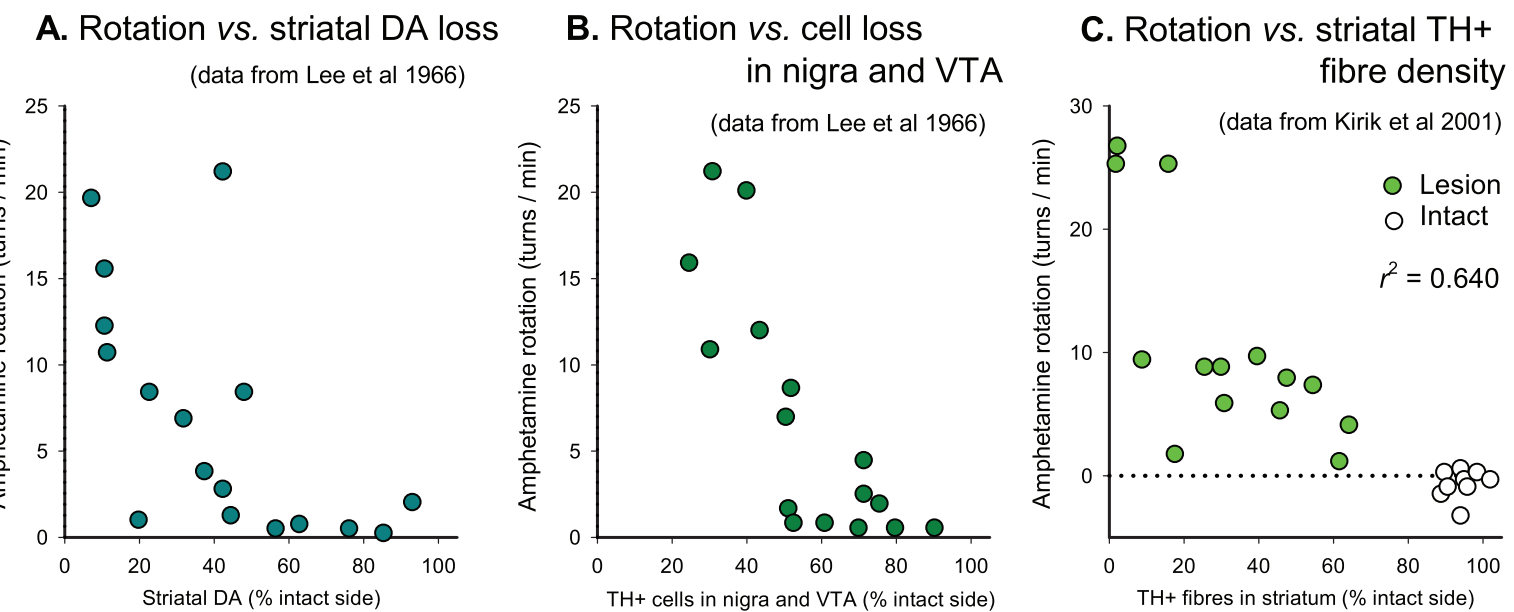

\section{6-OHDA lesions in MFB in rats \\ D. Rotation vs. striatal DA loss}

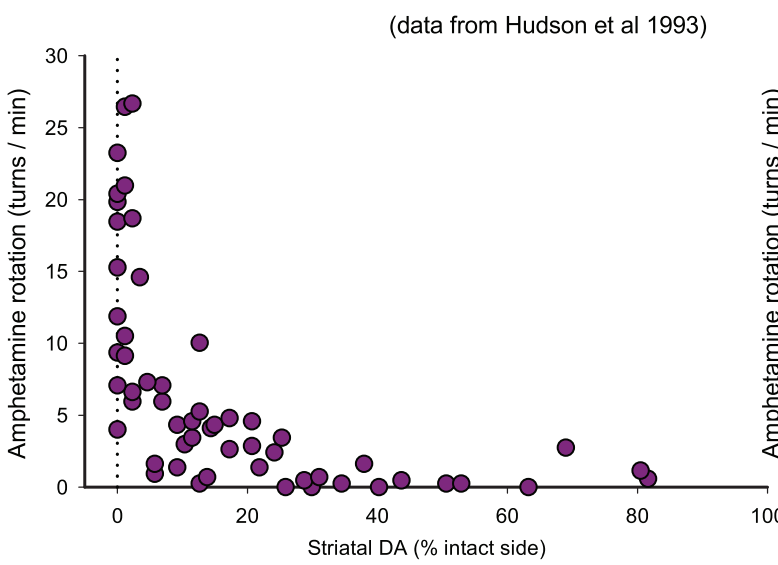

E. Rotation vs. nigral cell loss

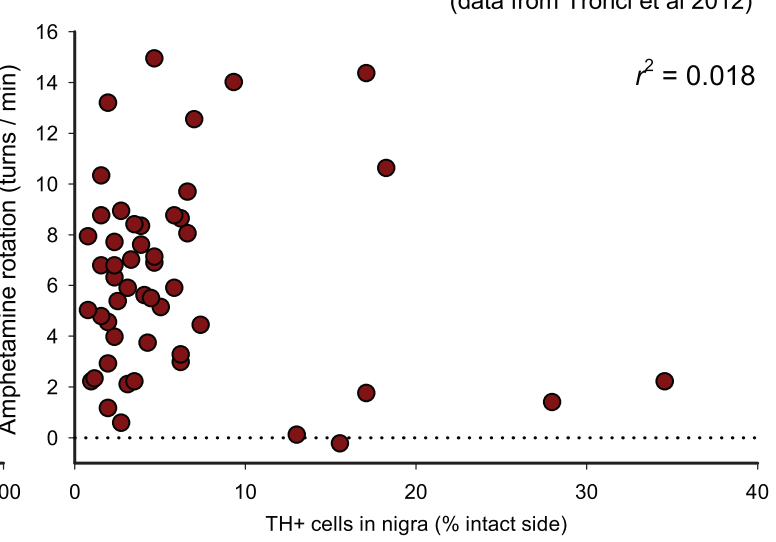

Fig. 1. AMPH-induced rotation in 6-OHDA lesioned rats. Scatter plots of turning rates of in rats with of 6-OHDA injections in the striatum (I) and MFB (II), expressed as mean $360^{\circ}$ turns $/ \mathrm{min}$, plotted against measures of striatal dopamine depletion (A, D), combined nigral and ventral tegmental $\mathrm{TH}+$ cell loss $(\mathrm{B})$, nigral $\mathrm{TH}+\operatorname{cell}$ loss $(\mathrm{E})$ or striatal $\mathrm{TH}+$ fibre density $(\mathrm{C})$. Dotted lines indicate the 0 turns/min rate of net behavioural symmetry between turning in lesion and intact directions. 6-OHDA, 6-hydroxydopamine; DA, dopamine concentration; MFB, medial forebrain bundle; $r^{2}$, Pearson's correlation coefficient (squared); TH, tyrosine hydroxylase; VTA, ventral tegmental area. Based on data obtained from Lee et al. [19], Hudson et al. [17], Kirik et al. [21], and Tronci et al. [18].

The non-linearity of the turning response is striking: in both rats and mice, and with either type of lesion, a loss of at least $40-50 \%$ of the nigral THpositive neurons, striatal TH-positive fibers, and striatal DA levels is needed before any significant turning starts to appear, and in animals with more severe lesions there is only a crude correlation between the rate of turning and the magnitude of cell loss or loss of striatal DA (see, e.g., Figs. 1D, E and 2A, B).

In MFB-lesioned rats and mice (Figs. 1D, E and $2 \mathrm{E}-\mathrm{H}$ ), high turning rates, $\geq 5$ turns/min in the rat and $\geq 3$ turns/min in mice, are typically seen in animals with $>80 \%$ loss of either nigral $\mathrm{TH}+$ neurons, striatal DA levels or $\mathrm{TH}+$ innervation in the dorsal striatum, but as shown by the data from Hudson et al. [17] and Tronci et al. [18], animals with such near-complete lesions can display very different turning rates, from 1-2 to over 20 turns/min (see Fig. 1D, E). As a rule, however, lower turning rates-below 5 turns/min in MFB-lesioned rats and below 4 turns/min in MFB-lesioned mice-are characteristic for lesions that range between 50 and $90 \%$. As seen from the data in (Figs. 1 and 2), turning rates below this threshold do not correlate with lesion size. 
I. Intrastriatal lesions

A. Rotation vs.nigral cell loss

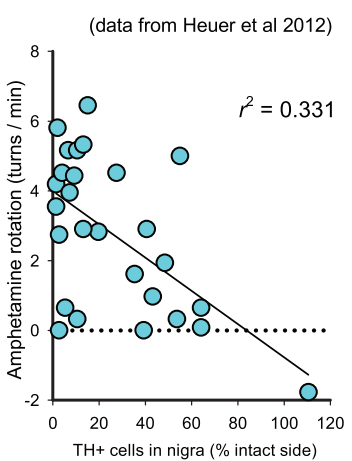

III. MFB lesions in mice

E. Rotation vs. nigral cell loss

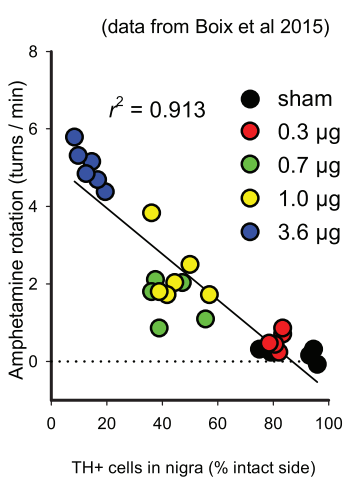

II. Intranigral lesions in mice

B. Rotation vs. nigral cell loss
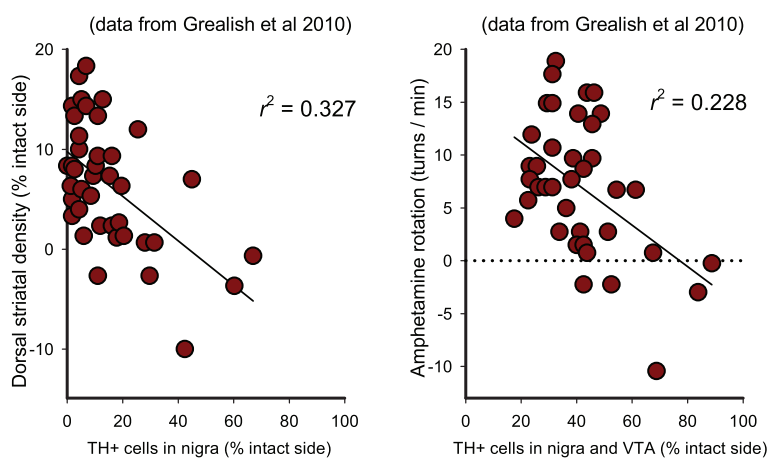

G. Rotation vs. ventral striatal denervation

(data from Boix et al 2015)

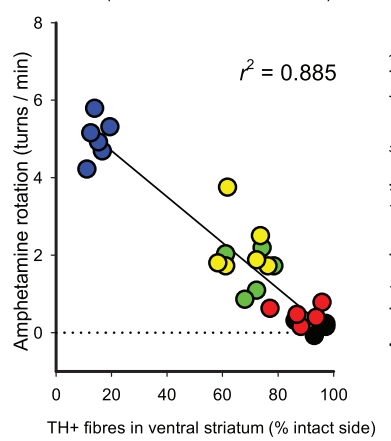

D. Rotation vs. striatal denervation

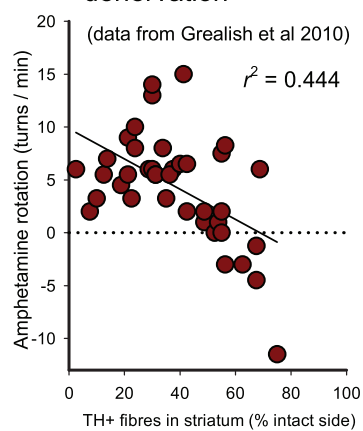

H. Rotation vs. nigral (data from Heuer et al 2012)
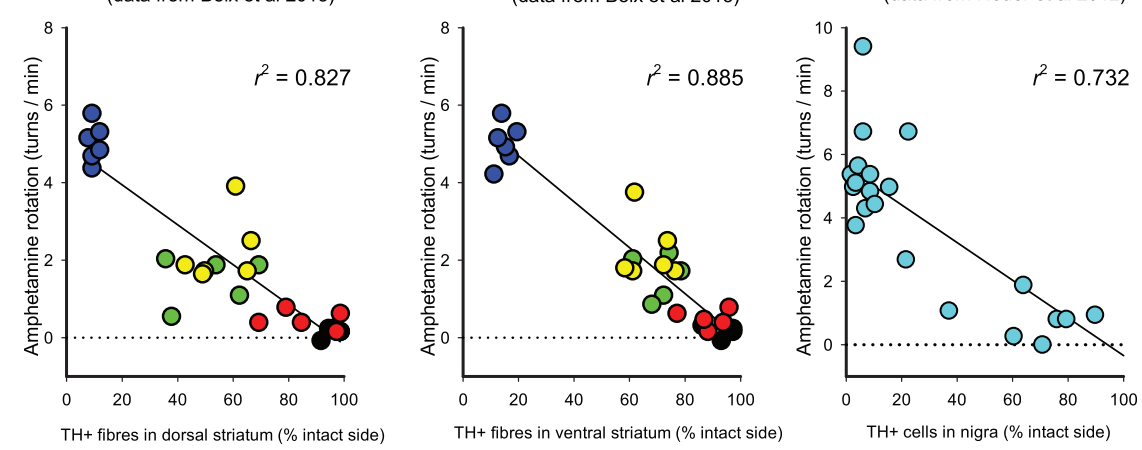

Fig. 2. AMPH-induced rotation in 6-OHDA lesioned mice. Scatter plots of turning rates of in rats with of 6-OHDA injections in the striatum (I), the substantia nigra (II) and MFB (III), expressed as mean $360^{\circ}$ turns/min, plotted against measures of nigral TH + cell loss (A, B, E, H), combined nigral and ventral tegmental cell loss $(\mathrm{C})$ and striatal $\mathrm{TH}+$ fibre density $(\mathrm{D}, \mathrm{F}, \mathrm{G})$. Dotted lines indicate the 0 turns/min rate of net behavioural symmetry between turning in lesion and intact directions. 6-OHDA, 6-hydroxydopamine; MFB, medial forebrain bundle; $r^{2}$, Pearson's correlation coefficient (squared); TH, tyrosine hydroxylase; VM, ventral mesencephalon; VTA, ventral tegmental area. Based on data obtained from Grealish et al. [10], Heuer et al. [20], and Boix et al. [44].

In animals with intrastriatal (Fig. $1 \mathrm{~A}-\mathrm{C}$ and Fig. 2A) or intranigral (Fig. 2B-D) lesions, the rate of turning is even less informative. In the most comprehensive studies performed so far [10, 19-21] turning rates in the range of 5-20 turns/min are seen in rats with lesions that span from about 50 to over $90 \%$ (Fig. 1A-C), and in mice lesions larger than 50-60\% show rather poor correlations with the turning rate (Fig. 2A-D). The reason for this mismatch is that the denervation caused by intrastriatal or intranigral 6-OHDA injections varies in both size and extent of the denervated areas within the striatum. It is well known that the striatum in rodents is functionally heterogenous, and that different sectors sub-serve different aspects of motor functions. Data from cell transplantation studies indicate that it is the DA innervation in the dorso-lateral part of the caudate-putamen, in particular, that drives turning behavior [22-24]. It is likely, therefore, that the rate of turning in response to amphetamine is determined by the extent of sparing of TH-positive fibres within this subregion. Due to small differences in the location and extent of the 6-OHDA lesion it is clear that this may vary from animal to animal independent of the overall magnitude of striatal denervation and nigral cell loss.

\section{The role of amphetamine acting outside the caudate-putamen}

Systemically administered amphetamine will stimulate DA release not only in the caudate-putamen but also in other DA-innervated areas involved in motor control, nucleus accumbens in particular. DA released 
in nucleus accumbens is known to induce locomotor behavior, and Kelly and More [25] have proposed a model for how the two areas interact. According to this model DA released in the caudate-putamen governs the direction of turning, whereas DA release in nucleus accumbens determines the turning rate. The role of nucleus accumbens as an amplifier of the turning response is supported by experiments performed by Kelly and Moore [26] and Brundin et al. [27], showing that disruption of accumbens function, either by lesioning or DA receptor blockade, attenuates the AMPH-induced turning response. It should be pointed out however, that the accumbens lesions used in these experiments were applied bilaterally, and that it is likely that denervation restricted to only one side of the brain will have much less effect. Nevertheless, it seems possible that differences in turning rates between intrastriatal and MFB lesions could be explained by this mechanism. Thus, the marked differences in turning rates in rats with intrastriatal or MFB lesions, seen at intermediate levels of DA neuron cell loss (50-80\%) (5-10 vs. 0-2 turns/min; see Fig. 1A, B, E), may be due to the fact that the MFB lesion impacts also the nucleus accumbens, in contrast to the intrastriatal lesion where this area is left intact.

\section{Selection of the lesion placement}

From the above, it is apparent that the site of 6OHDA injection will influence the pattern and extent of DA cell loss and areas of forebrain denervation. $M F B$ lesions offer the possibility to obtain nearcomplete loss of nigral DA neurons and a 95-99\% loss of neostriatal DA innervation. Such lesions are the most reliable to produce high rates of rotation, typically a mean of 6-20 turns per minute, but are in most cases also accompanied by $30-70 \%$ denervation of the ventral striatum/nucleus accumbens and other forebrain targets. MFB lesions, however, are challenging to use in mice due to the small size of their brains and a high mortality rate. For experiments in mice, therefore, lesions targeting the substantia nigra itself, as used in the Grealish et al. study [9, 10], is a realistic alternative, but given the small size and deep location of this nucleus to the ventral surface of the brain, accurate and reproducible lesions may be difficult to achieve, even in skilled surgical hands. Intrastriatal 6-OHDA lesions are commonly used in both rats and mice. In contrast to MFB lesions, however, the intrastriatal lesions will affect only a subset of nigral/VTA neurons and the functional impact will depend on the extent and location of the denervation within the striatum. It is important to keep in mind that the rate of AMPH rotation reflects primarily the extent of denervation in the dorsolateral part of the caudate-putamen. For this reason, AMPH-induced rotation cannot be used as a stand-alone test to assess the magnitude of cell loss in the substantia nigra-it will depend on the subset of neurons and the area of the striatum affected by the lesion.

\section{$A M P H$ - and $A P O$-induced rotation are poorly correlated}

These two measures are often used in parallel assuming that they measure the same thing. Due to their different modes of action however, the turning behaviors driven by these two drugs reflect activation of different signaling mechanisms and pathways, and are not equivalent. There are ample examples of the degree of mismatch between AMPH and APO rotation scores in the literature. In (Fig. 3), we illustrate this point with a summary of the rotation data published by Heuer et al. [20] which were collected from over 100 mice subjected to 6-OHDA lesions placed either in the striatum, substantia nigra or MFB. As evident from panel 1A, AMPH and APO rotation scores are poorly correlated at the level of the individual mouse. On a group level, however, both measures are significantly correlated with the extent of nigral cell loss although the overall correlation is better for AMPH-induced rotation scores $(r=0.755$; Fig. $3 \mathrm{~B})$ than for APO-induced scores $(r=0.575$; Fig. 3C). From a closer inspection of the scatter plots it is clear that AMPH rotation is a more reliable measure to identify animals with more severe lesions $(>50-60 \%)$ of the nigral DA projection. Although high APO-induced turning rates are typical for mice with lesions that exceed $80 \%$, APO-induced rotation is less useful as a tool to identify well-lesioned animals. An additional disadvantage of the APO test is that it is sensitive to non-specific damage to the striatum, induced, e.g., by surgical interventions [15, 28]. Such damage is known to cause a reduction in the turning rate and can thus lead to misinterpretation of the collected data (see [28] for further discussion).

\section{AMPH rotation as a predictor of impairment in motor behavior}

The rotation test is a quick and convenient experimental tool and it is for that reason important to know its predictive value, i.e., to what extent can we 
A. Amphetamine vs. Apomorphine

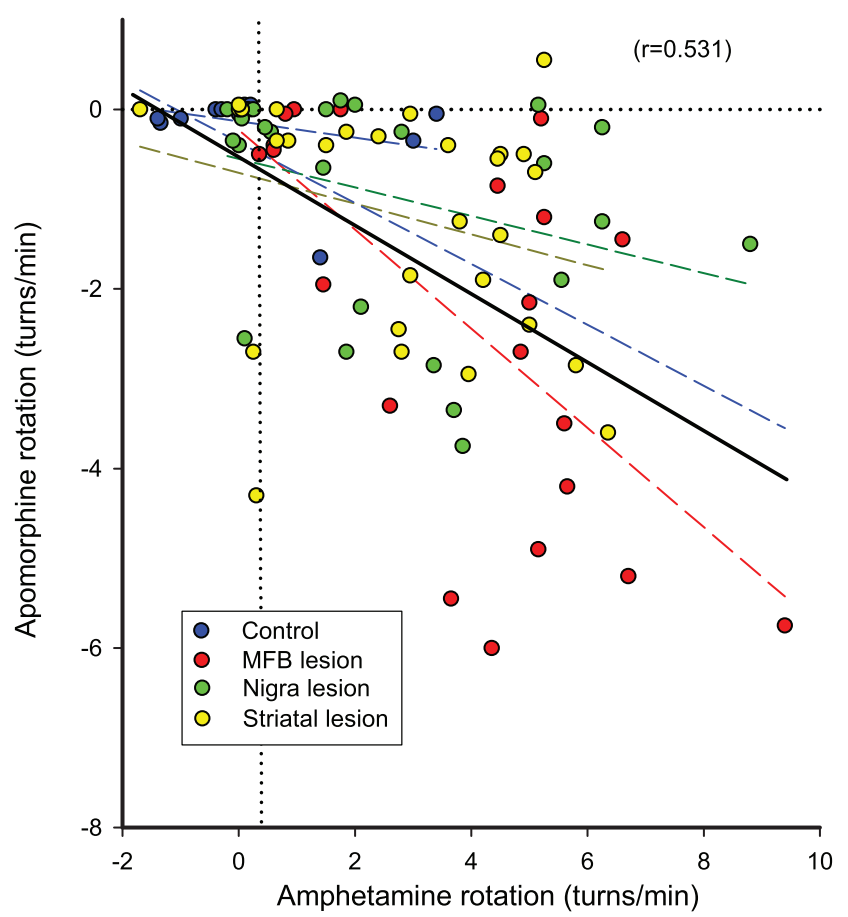

B. Cell loss vs. Amphetamine

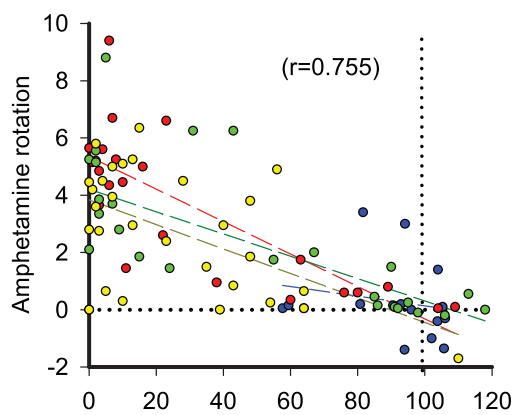

C. Cell loss vs. Apomorphine

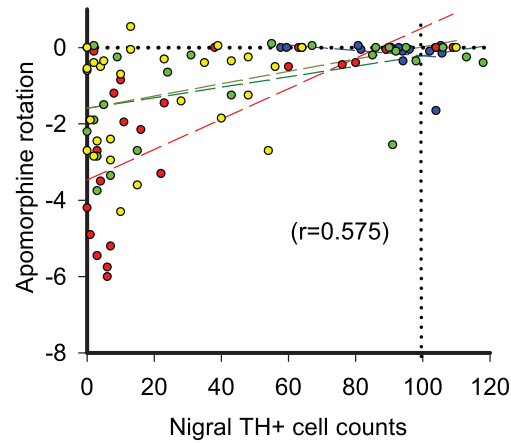

Fig. 3. Correlations between AMPH and APO turning rates in groups of control mice and mice with 6-OHDA injected into three different (striatal, MFB or nigral) targets (A). AMPH-induced turning rates correlate better with nigral TH+cell loss (B), than APO-induced turning rates $(\mathrm{C})$. The 'best fit' regression lines were calculated by parametric analysis both for the separate groups and for all animals combined, and the Pearson correlation coefficient is shown as $r^{2}$ to indicate the proportion of variance accounted for in the combined group analyses. MFB, medial forebrain bundle; TH, tyrosine hydroxylase. Unpublished data reanalyzed from the study by Heuer et al. [20], with thanks to Dr. Andreas Heuer for supply of the original data files.

rely on this single test as a measure of impairment in motor behavior? On a group level the correlation between high rates of AMPH turning, $\geq 5$ turns/min in rats and $\geq 3$ turns/min in mice, and impairment in standard tests of spontaneous motor behavior is usually quite good. In our own studies most, but not all, rats displaying such high turning rates show marked impairments in stepping and paw use in the cylinder and staircase tests [21] and most mice with high AMPH-induced turning rates show significant impairments in some but not all spontaneous motor tests $[10,20,29]$. In the Grealish et al. [10] study the so-called corridor test, which monitors the sensorimotor attention deficit on the side opposite to the lesion [30], was the one that distinguished most accurately between lesions of different severity. In (Fig. 4), the performances of mice with severe (80-100\% striatal denervation), intermediate (60-79\% denervation) and mild ( $<60 \%$ denervation) lesions are compared for the corridor, stepping and cylinder tests, in comparison with the average scores obtained in the
AMPH and APO rotation tests. High rates of turning in response to either AMPH or APO predict marked deficits in the corridor test, but it is notable that the AMPH scores, in this experiment, did not distinguish between animals with severe and intermediate size lesions. The stepping and cylinder tests, which are highly useful to assess motor deficits in unilaterally lesioned rats, show some variability between studies in mice $[10,20]$.

\section{FUNCTIONAL RECOVERY IN 6-OHDA LESIONED RATS AND MICE}

AMPH-induced rotation is commonly used as a practical and convenient test of functional recovery in cell transplantation and neuroprotection studies. It is highly useful for this purpose and also very sensitive; complete reversal of AMPH rotation is seen in animals with transplants containing less than 500 surviving DA neurons (Fig. 5A) and obtained with 


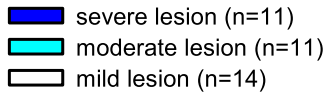

B. APO rotation
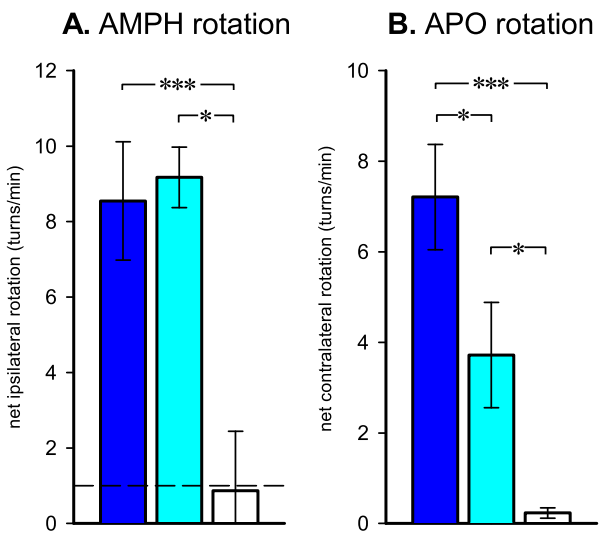

\section{Corridor test}

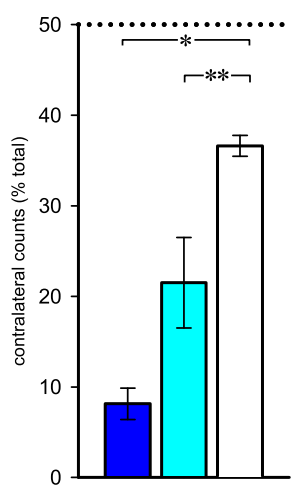

.... no side bias

- control level (unlesioned mice)
D. Stepping test

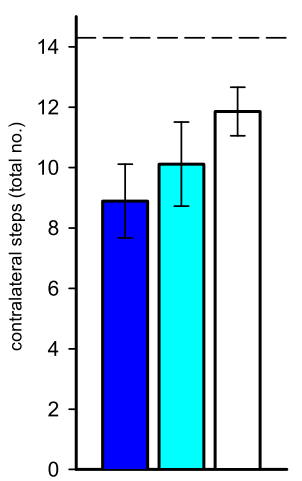

E. Cylinder test

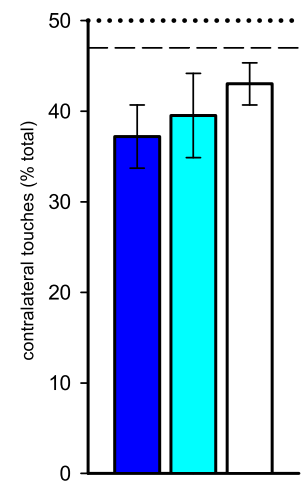

Fig. 4. The capacity of AMPH- and APO-induced rotation (A and B) and three commonly used tests of spontaneous motor behavior (C-E), to discriminate between lesions of different degrees of completeness in 6-OHDA lesioned mice. 36 mice were sub-classified as exhibiting severe $(80-100 \%)$, intermediate $(60-79 \%)$, or mild $(<60 \%)$ lesions based on the magnitude of $\mathrm{TH}+$ cell loss in the ipsilateral substantia nigra. The corresponding magnitude of functional deficit was progressive and highly significant for AMPH-rotation, APO-rotation and the corridor task, but failed to exhibit clear significant differences in the stepping and cylinder tests. ${ }^{*} p<0.05 ;{ }^{* *} p<0.001 ;{ }^{* * *} p<0.0001$. Data redrawn from Grealish et al. [10].

grafts that restore as little as $5 \%$ of the striatal DA content (Fig. 5B). However, this test has important limitations and pitfalls that may limit the conclusions that can be drawn from AMPH rotation data:

First, as illustrated in (Fig. 5A, B), recovery in AMPH rotation is not linear. The test is very sensitive but it cannot be used to determine either graft size or extent of striatal reinnervation. Thus, differences in turning rate beyond a threshold level (around 5 turns/min in rats, and 3 turns/min in mice) have little informative value. For this reason, changes within this range, say from 20 to 6 turns/min, cannot be interpreted as a sign of DA-mediated functional recovery. For the same reason it is misleading to present changes in AMPH rotation as percent reduction in the rotation scores; AMPH rotation data should always be reported as turns/min.

Second, overcompensation, i.e., AMPH induced rotation in the direction away from the lesioned and grafted side, is commonly seen in animals with functional DA neuron grafts. The precise mechanism of overcompensation is not clear, but it is manifestly not due to the grafts providing an excess of dopamine neurons or striatal DA levels above normal (Fig. 5A, B, respectively). Rather, we must consider a complex interaction between partial graft-derived reinnervation of the denervated striatum, the extent of spread of amphetamine-stimulated dopamine release through the striatum, and the degree of restoration of postsynaptic receptor supersensitivity to normal levels within proximal and distal areas within the whole dorsal and ventral striatum. The overall rate of contralateral turning is unrelated to graft size or extent of $\mathrm{TH}+$ fibre outgrowth and should for this reason be interpreted with caution. However, the time-course of the contralateral response has been found to be crudely related to graft size: in rats with relatively small grafts contralateral turning tend to be limited to the first 15-20 min after AMPH injection, whereas in rats with larger grafts contralateral rotation continues over the entire 90 min observation period, suggesting that DA terminal storage and buffering capacity is a factor in the maintenance of contralateral turning over time [31, 32]. Distinctive differences in the time course of the overcompensation response, i.e., the size of the early peak and the duration of the contralateral response, may be possible to use as a crude measure of the size of the graft $[33,34]$.

Third, recovery in the AMPH rotation test does not always signify recovery of spontaneous motor or sensorimotor behavior. In cell transplant experiments complete reversal in the AMPH rotation test can be obtained with transplants that are too small to have any impact in the standard tests of spontaneous motor behavior, and with larger size transplants complete reversal or overcompensation in the AMPH test is, at best, associated with partial recovery in test of spontaneous motor function (Fig. 5C). It is important 
A. Amphetamine rotation vs. $\mathrm{TH}+$ cells in grafts

(data from Brundin et al 1988)

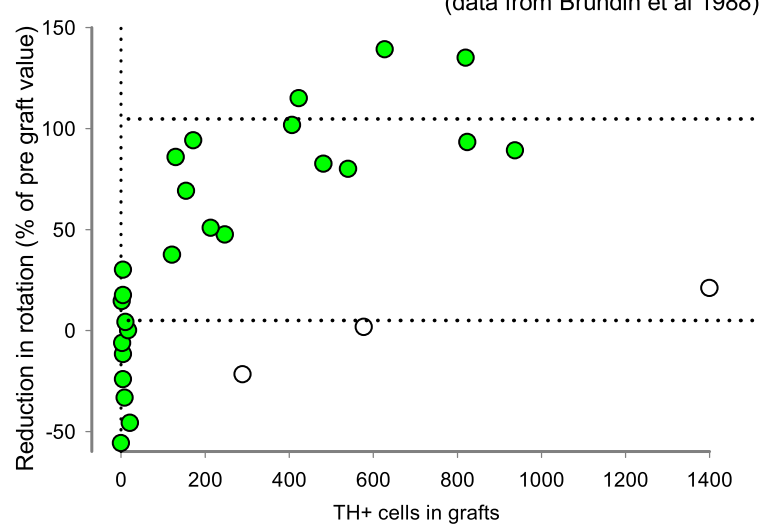

C. Comparison of recovery in 4 tests
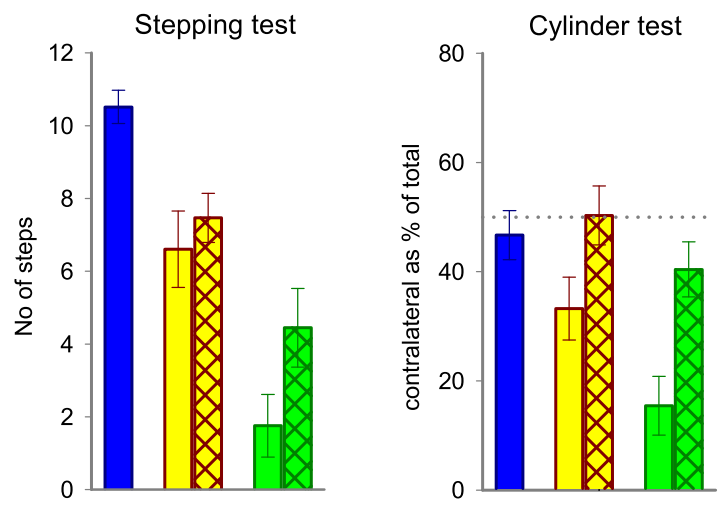

(data from Kirik et al 2001)

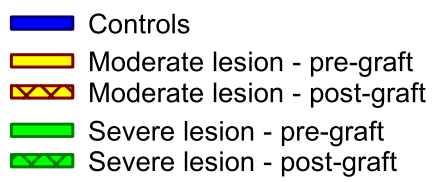

\section{B. Amphetamine rotation vs. striatal DA content}
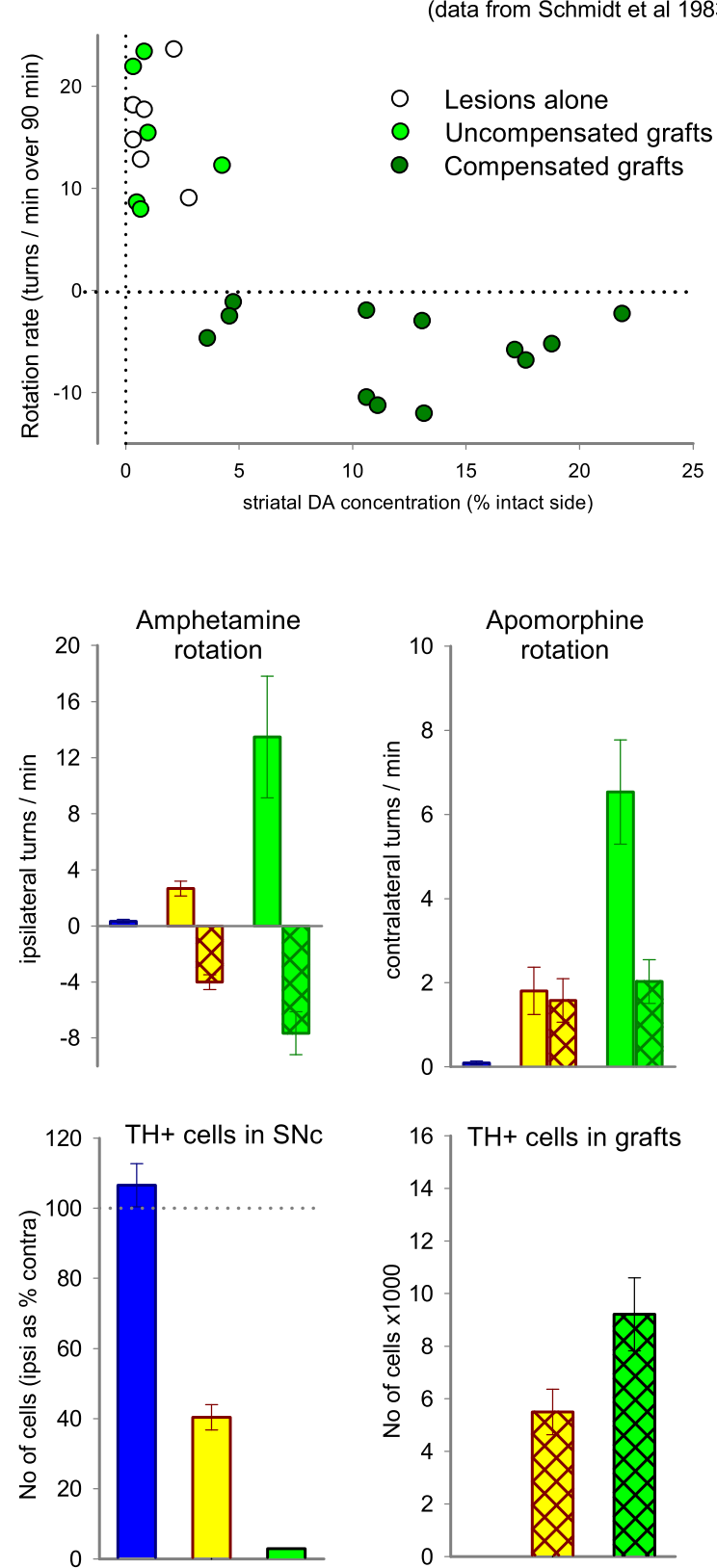

Fig. 5. AMPH-induced rotation as a tool to monitor functional recovery in 6-OHDA leisoned rats. In rats with lesions of the MFB the degree of compensation of rotational asymmetry induced by intrastriatal VM cell suspension grafts matches well the number of surviving DA neurons (A) and striatal DA concentration (B), although the correlation is not linear. In both experiments a proportion of animals exhibit 'overcompensation', i.e., recovery to a degree that the ipsilateral turning deficit induced by the lesions is restored to a modest contralateral bias. (C) Recovery following intrastriatal VM grafts in rats with intrastriatal 6-OHDA lesions, showing post-lesion (pre-graft) deficits and post-graft recovery on a range of behavioural tests. Animals are subdivided according to whether they had partial (moderate) or relatively complete (severe) lesions based on the magnitude of $\mathrm{TH}+$ cell loss in the substantia nigra. $\mathrm{TH}$, tyrosine hydroxylase; SNc, substantia nigra pars compacta. Based on data obtained from Brundin et al. [45], Schmidt et al. [46], and Kirik et al. [21]. 
to keep in mind that recovery in spontaneous motor function, as seen, e.g., in the stepping and cylinder tests, is obtained with DA neuron-rich transplants that provide more extensive striatal reinnervation of the denervated striatum that goes well beyond what is needed to reverse the AMPH rotation deficit.

Fourth, the status of the DA innervation of the ventral striatum may play a role, not only as amplifier of the AMPH turning rate, as mentioned above, but also in determining the extent of functional recovery induced by transplants innervating the dorsal striatum. Thus, Breysse et al. [35] have shown that recovery in paw use (in this case the stepping test) induced by intrastriatal DA neuron grafts is in part dependent on the integrity of the innervation of the ventral striatum.

Fifth, behavioral conditioning and habituation may be a confounding factor. Rotational asymmetry is typically considered to be a relatively pure test of imbalances in the physiology of the two striata, but pharmacological and behavioural factors can influence performance. Even normal rats can develop individual biases, in part due to underlying asymmetries in nigrostriatal dopamine function [36]. Animals can develop habitual behaviours through repetition and reward-related learning, which will also influence underlying dopamine turnover [37, 38]. In addition to variability of lesion magnitude, the repetition and frequency of post-lesion testing will influence observed rotation rates. Thus, in the AMPH rotation test the animals will sensitize, i.e., exhibit progressively higher rates of turning with repeated testing [39], whereas apomorphine rotation can reduce with frequently repeated dosing by dopamine agonists, associated with a reversal of the lesion-induced receptor supersensitivity [40, 41]. Finally, in transplanted animals, there is now ample evidence that training and experience modifies the functional integration of the transplants [42]. Consequently, however useful as a convenient metric of behavioural deficit and functional recovery, changes in AMPH rotation do not necessarily provide a straightforward index of the precise level of recovery and repair.

\section{SUMMARY AND RECOMMENDATIONS}

Based on the considerations discussed above, and in order to avoid misinterpretations of AMPH rotation data, we recommend the user of the AMPH rotation test to adhere to the following basic rules.
1. Rotation data should be collected over the entire measuring period (usually $90 \mathrm{~min}$ in rats and $40 \mathrm{~min}$ in mice) and reported as mean net full $360^{\circ}$ turns/minute (ipsilateral minus contralateral turns), using a standard dose of $\mathrm{d}$-amphetamine, in the range of $2.5-5.0 \mathrm{mg} / \mathrm{kg}$, typically administered i.p. [3, 43]. Recordings spanning over the entire response period is now the accepted standard in the field, used in order to avoid variability due to differences in the pharmacokinetics (i.e., time-course of the action of the drug), or DA release kinetics, between animals. Note that d-amphetamine is the biologically active isomer, the l-isomer is inactive. Thus, a racemic d, 1 mixture has half the potency (i.e. double dosage). Methamphetamine is a more potent variant and can yield higher rates of rotation, but at the risk of overdosing and loss of animals during testing. For these reasons we recommend the d-amphetamine for rotation testing. Note, moreover, that in most countries, amphetamines are a restricted drug class and will require regulatory approval before purchase and use.

2. For studies of changes in AMPH rotation over time, such as used in experiments aimed at functional recovery or neuroprotection, it is essential that the experimental groups are well balanced. We recommend two consecutive pre-intervention tests, performed with about one week's interval, to establish the baseline rotation score used to match animals into different experimental groups. The baseline scores should be taken not earlier than 2 weeks after the lesion, i.e., at a time when the rotation rate has stabilized.

3. Lesioned rats and mice used in experiments aimed to assess graft-induced functional recovery should be pre-selected based on a baseline score of $\geq 5$ turns $/ \mathrm{min}$ in rats and $\geq 3$ turns $/ \mathrm{min}$ in mice. As discussed above, this criterion will identify MFB-lesioned animals with $\mathrm{TH}-$ positive nigral cell loss that exceeds $80 \%$, and intrastriatally lesioned animals with a $\mathrm{TH}-$ positive nigral cell loss ranging from 50 to $90 \%$. Since lower turning rates tend to be unstable and may recover spontaneously over time, this pre-selection will reduce variability within the groups and also help to avoid inclusion of false positives.

4. To assess the extent of lesion-induced nigral TH-positive cell loss and the extent of 
functional recovery in individual rats or mice, it is necessary to combine the AMPH rotation test with one or several tests of spontaneous motor behavior. For 6-OHDA lesioned rats we recommend paw use in the cylinder test, performed as described, e.g., in the review of Winkler et al. [1], and for 6-OHDA lesioned mice lateralized sensorimotor behavior in the corridor test, performed as described by Dowd et al. [30]. These two tests are easy to apply, recorded from videos that can be scored by a blind rater, and do not require specially trained personnel.

5. APO-induced rotation reflects changes in postsynaptic sensitivity and can thus only be an indirect measure of loss or recovery of striatal DA neurotransmission. It should be administered at a low dose, $0.05-0.1 \mathrm{mg} / \mathrm{kg}$ in rats, in order to target supersensitive receptors exclusively, and is commonly administered s.c. Higher doses, in the range $0.5-4 \mathrm{mg} / \mathrm{kg}$, s.c., are required for mice in order to induce turning, and may benefit from 2 priming injections over the preceding days to avoid any potential wind-up effect [10]. It is important to keep in mind that APO rotation can diminish in response to nonspecific striatal damage, unrelated to the status of the damaged dopamine pathways. Consequently, whereas APO rotation can provide a useful adjunct to the AMPH rotation test, it should not be used alone as the sole index of DA neuron loss, recovery or repair.

6. In studies of functional recovery, or neuroprotection aimed at functional sparing, it is essential that the rotation scores are reported as actual full $360^{\circ}$ turns/minute, and not as \% change. The reason for this is that the AMPH turning response is far from linear (see above). Thus, it is important to keep in mind that partial reductions in AMPH turning above the critical threshold, say from 20 to 6 turns/minute, is not possible to interpret as a sign of functional recovery.

7. Results should be reported both as changes in turning rate over time in the experimental and control groups, as well as the difference between groups at different time points. Since repeated amphetamine (and apomorphine) treatment can show sensitization, i.e., an increase in rotational score in repeated tests evidence of functional recovery induced by the experimental intervention (e.g., a cell transplant) cannot be based exclusively on a comparison between groups at single time points. If the control group shows increased rotation over time, then the difference relative to the experimental group may be erroneously interpreted as a sign of functional recovery.

8. Rotation tests may be repeated at regular intervals, but frequent closely spaced tests should be avoided. Two post treatment rotation tests should be separated by at least 1 -week interval off drug. For longitudinal tests once per month provides a good compromise.

\section{ACKNOWLEDGMENTS}

We thank Dr. Andreas Heuer for the provision of raw data used in generating Fig. 3, and to both him and Dr. Shane Grealish for valuable suggestions on the draft manuscript.

\section{CONFLICT OF INTEREST}

The authors have no conflict of interest to report.

\section{REFERENCES}

[1] Winkler C, Kirik D, Björklund A, Dunnett SB (2000) Transplantation in the rat model of Parkinson's disease: Ectopic versus homotopic graft placement. Prog Brain Res 127, 233-265.

[2] Dunnett SB (2005) Motor functions of the nigrostriatal dopamine system: Studies of lesions and behaviour. In Handbook of Chemical Neuroanatomy. Vol. 21. Dopamine, Dunnett SB, Bentivoglio M, Björklund A, Hökfelt T, eds. Elsevier, Amsterdam \& New York, pp. 235-299.

[3] Dunnett SB, Torres EM (2011) Rotation in the 6-OHDA lesioned rat. In Animal Models of Movement Disorders: Volume I. Neuromethods vol. 61, Lane EL, Dunnett SB, eds. Springer/Humana, New York, pp. 299-315.

[4] Andén N-E, Carlsson A, Dahlström A, Fuxe K, Hillarp NA, Larsson K (1964) Demonstration and mapping out of nigroneostriatal dopamine neurons. Life Sci 3, 523-430.

[5] Anden NE, Dahlstrom A, Fuxe K, Larsson K (1965) Mapping out of catecholamine and 5-hydroxytryptamine neurons innervating the telencephalon and diencephalon. Life Sci 4, 1275-1279.

[6] Andén N-E, Dahlström A, Fuxe K, Larsson K (1966) Functional role of the nigro-neostriatal dopamine neurons. Acta Pharmacol Toxicol 24, 263-274.

[7] Ungerstedt U, Arbuthnott GW (1970) Quantitative recording of rotational behaviour in rats after 6-hydroxydopamine lesions of the nigrostriatal dopamine system. Brain Res 24, 485-493.

[8] Schwarting RKW, Huston JP (1996) Unilateral 6hydroxydopamine lesions of meso-striatal dopamine neurons and their physiological sequelae. Prog Neurobiol 49, 215-266.

[9] Pycock C, Tarsy D, Marsden CD (1975) Inhibition of circling behavior by neuroleptic drugs in mice with unilateral 
6-hydroxydopamine lesions of the striatum. Psychopharmacologia 45, 211-219.

[10] Grealish S, Mattsson B, Draxler P, Björklund A (2010) Characterisation of behavioural and neurodegenerative changes induced by intranigral 6-hydroxydopamine lesions in a mouse model of Parkinson's disease. Eur J Neurosci 31, 2266-2278.

[11] Ulusoy A, Decressac M, Kirik D, Björklund A (2010) Viral vector-mediated overexpression of alpha-synuclein as a progressive model of Parkinson's disease. Prog Brain Res 184, 89-111.

[12] Volpicelli-Daley LA, Kirik D, Stoyka LE, Standaert DG, Harms AS (2016) How can rAAV-alpha-synuclein and the fibril alpha-synuclein models advance our understanding of Parkinson's disease? J Neurochem 139(Suppl 1), 131-155.

[13] Ungerstedt U (1971) Striatal dopamine release after amphetamine or nerve degeneration revealed by rotational behaviour. Acta Physiol Scand Suppl 367, 49-68.

[14] Ungerstedt U (1971) Postsynaptic supersensitivity after 6-hydroxydopamine-induced degeneration of the nigrostriatal dopamine system. Acta Physiol Scand Suppl 367, 69-93.

[15] Marshall JF, Ungerstedt U (1977) Striatal efferent fibers play a role in maintaining rotational behavior in the rat. Science 198, 62-64.

[16] Koshikawa N (1994) Role of the nucleus accumbens and the striatum in the production of turning behaviour in intact rats. Rev Neurosci 5, 331-346.

[17] Hudson JL, van Horne CG, Strömberg I, Brock S, Clayton J, Masserano J, Hoffer BJ, Gerhardt GA (1993) Correlation of apomorphine- and amphetamine-induced turning with nigrostriatal dopamine content in unilateral 6-hydroxydopamine lesioned rats. Brain Res 626, 167-174.

[18] Tronci E, Shin E, Bjorklund A, Carta M (2012) Amphetamine-induced rotation and 1-DOPA-induced dyskinesia in the rat 6-OHDA model: A Correlation study. Neurosci Res 73, 168-172.

[19] Lee CS, Sauer H, Björklund A (1996) Dopaminergic neuronal degeneration and motor impairments following axon terminal lesion by intrastriatal 6-hydroxydopamine in the rat. Neuroscience 72, 641-653.

[20] Heuer A, Smith GA, Lelos MJ, Lane EL, Dunnett SB (2012) Unilateral nigrostriatal 6-hydroxydopamine lesions in mice. I. Behavioural assessment of unilateral striatal dopamine depletion via three different lesion sites. Behav Brain Res 228, 30-43.

[21] Kirik D, Winkler C, Björklund A (2001) Growth and functional efficacy of intrastriatal nigral transplants depends on the extent of nigrostriatal degeneration. J Neurosci 21, 28892896.

[22] Dunnett SB, Björklund A, Stenevi U, Iversen SD (1981) Grafts of embryonic substantia nigra reinnervating the ventrolateral striatum ameliorate sensorimotor impairments and akinesia in rats with 6-OHDA lesions of the nigrostriatal pathway. Brain Res 229, 209-217.

[23] Dunnett SB, Björklund A, Stenevi U, Iversen SD (1981) Behavioral recovery following transplantation of substantia nigra in rats subjected to 6-OHDA lesions of the nigrostriatal pathway. 1. Unilateral lesions. Brain Res 215, 147-161.

[24] Dunnett SB, Björklund A, Schmidt RH, Stenevi U, Iversen SD (1983) Intracerebral grafting of neuronal cell suspensions. IV. Behavioral recovery in rats with unilateral 6-OHDA lesions following implantation of nigral cell suspensions in different forebrain sites. Acta Physiol Scand Suppl 522, 29-37.
[25] Kelly PH, Moore KE (1976) Mesolimbic dopaminergic neurones in the rotational model of nigrostriatal function. Nature 263, 695-696.

[26] Kelly PH, Moore KE (1977) Mesolimbic dopamine neurons: Effects of 6-hydroxydopamine-induced destruction and receptor blockade on drug-induced rotation of rats. Psychopharmacology 55, 35-41.

[27] Brundin P, Strecker RE, Londos E, Björklund A (1987) Dopamine neurons grafted unilaterally to the nucleus accumbens affect drug-induced circling and locomotion. Exp Brain Res 69, 183-194.

[28] Barker RA, Dunnett SB (1994) Ibotenic acid lesions of the striatum reduce drug-induced rotation in the 6-hydroxydopamine-lesioned rat. Exp Brain Res 101, 365-374.

[29] Heuer A, Vinh NN, Dunnett SB (2013) Behavioural recovery on simple and complex tasks by means of cell replacement therapy in unilateral 6-hydroxydopaminelesioned mice. Eur J Neurosci 37, 1691-1704.

[30] Dowd E, Monville C, Torres EM, Dunnett SB (2005) The corridor task: A Simple test of lateralised response selection and neglect sensitive to unilateral dopamine deafferentation and graft-derived replacement in the striatum. Brain Res Bull 68, 24-30.

[31] Brundin P, Duan WM, Sauer H (1994) Functional effects of mesencephalic dopamine neurons and adrenal chromaffin cells grafted to the rodent striatum. In Functional Neural Transplantation, Dunnett SB, Björklund A, eds. Raven Press, New York, pp. 9-46.

[32] Rioux L, Gaudin DP, Bui LK, Grégoire L, DiPaolo T, Bédard PJ (1991) Correlation of functional recovery after a 6-hydroxydopamine lesion with survival of grafted fetal neurons and release of dopamine in the striatum of the rat. Neuroscience 40, 123-131.

[33] Herman JP, Rouge-Pont F, Le Moal M, Abrous DN (1993) Mechanisms of amphetamine-induced rotation in rats with unilateral intrastriatal grafts of embryonic dopaminergic neurons: A Pharmacological and biochemical analysis. Neuroscience 53, 1083-1095.

[34] Torres EM, Dunnett SB (2007) Amphetamine induced rotations in the assessment of lesions and grafts in the unilateral rat model of Parkinson's disease. Eur Neuropsychopharmacol 17, 206-214.

[35] Breysse N, Carlsson T, Winkler C, Björklund A, Kirik D (2007) The functional impact of the intrastriatal dopamine neuron grafts in parkinsonian rats is reduced with advancing disease. J Neurosci 27, 5849-5856.

[36] Glick SD, Jerussi TP, Water DH, Green JP (1974) Amphetamine-induced changes in striatal dopamine and acetylcholine levels and relationship to rotation (circling behavior) in rats. Biochem Pharmacol 23, 3223-3225.

[37] Dunnett SB, Björklund A (1983) Conditioned turning in rats: Dopaminergic involvement in the initiation of movement rather than the movement itself. Neurosci Lett 41, 173-178.

[38] Freed CR, Yamamoto BK (1985) Regional brain dopamine metabolism: A Marker for the speed, direction, and posture of moving animals. Science 229, 62-65.

[39] Robinson TE(1984) Behavioral sensitization: Characterization of enduring changes in rotational behavior produced by intermittent injections of amphetamine in male and female rats. Psychopharmacology 84, 466-475.

[40] Schneider MB, Murrin LC, Pfeiffer RF, Deupree JD (1984) Dopamine receptors: Effects of chronic L-dopa and 
bromocriptine treatment in an animal model of Parkinson's disease. Clin Neuropharmacol 7, 247-257.

[41] Winkler JD, Weiss B (1986) Reversal of supersensitive apomorphine-induced rotational behavior in mice by continuous exposure to apomorphine. Journal of Pharmacol Exp Ther 238, 242-247.

[42] Brasted PJ, Watts C, Robbins TW, Dunnett SB (1999) Associative plasticity in striatal transplants. Proc Natl Acad Sci U S A 96, 10524-10529.

[43] Smith GA, Heuer A (2011) 6-OHDA toxin models of PD in the mouse. In Animal Models of Movement Disorders: Volume I. Neuromethods vol. 61, Lane EL, Dunnett SB, eds. Springer/Humana, New York, pp. 281-297.
[44] Boix J, Padel T, Paul G (2015) A partial lesion model of Parkinson's disease in mice-characterization of a 6OHDA-induced medial forebrain bundle lesion. Behav Brain Res 284, 196-206.

[45] Brundin P, Barbin G, Strecker RE, Isacson O, Prochiantz A, Björklund A (1988) Survival and function of dissociated rat dopamine neurons grafted at different developmental stages or after being cultured in vitro. Dev Brain Res 467, 233-243.

[46] Schmidt RH, Björklund A, Stenevi U, Dunnett SB, Gage FH (1983) Intracerebral grafting of neuronal cell suspensions. III. Activity of intrastriatal nigral suspension implants as assessed by measurements of dopamine synthesis and metabolism. Acta Physiol Scand Suppl 522, 19-28. 